\title{
Identification of multi-input systems: variance analysis and input design issues
}

\author{
M. Gevers ${ }^{\mathrm{a}, 1}$ L. Mišković ${ }^{\mathrm{b}, 2}$ D. Bonvin ${ }^{\mathrm{b}}$ A. Karimi ${ }^{\mathrm{b}}$ \\ ${ }^{a}$ Center for Systems Engineering and Applied Mechanics (CESAME) \\ Université Catholique de Louvain \\ B-1348 Louvain-la-Neuve, Belgium \\ gevers@csam.ucl.ac.be \\ ${ }^{\mathrm{b}}$ Laboratoire d'Automatique \\ Ecole Polytechnique Fédérale de Lausanne (EPFL) \\ CH-1015 Lausanne, Switzerland \\ \{ljubisa.miskovic, dominique.bonvin, alireza.karimi\}@epfl.ch
}

\begin{abstract}
This paper examines the identification of multi-input systems. Motivated by an experiment design problem (should one excite the various inputs simultaneously or separately), we examine the effect of an additional input on the variance of the estimated coefficients of parametrized rational transfer function models, with special emphasis on the commonly used FIR, ARX, ARMAX, OE and BJ model structures. We first show that, for model structures that have common parameters in the input-output and noise models (e.g. ARMAX), any additional input contributes to a reduction of the covariance of all parameter estimates. We then show that the accuracy improvement extends beyond the case of common parameters in all transfer functions, and we show exactly which parameter estimates are improved when a new input is added. We also conclude that it is always better to excite all inputs simultaneously.
\end{abstract}

Key words: Identification, variance analysis, input design, multi-input systems

\footnotetext{
1 This paper presents research results of the Belgian Programme on Interuniversity Attraction Poles, initiated by the Belgian Federal Science Policy Office. The scientific responsibility rests with its authors.

2 The work of this author is supported by Swiss National Science Foundation under grant No 2100-66876.01
} 


\section{Introduction}

Our initial motivation for this piece of research was as follows. Suppose we are to identify a system with one output and several inputs, and we are given a limited amount of experimentation time to do so. Is it then better to excite one input at a time, each over part of the experimentation time, or should one excite all inputs simultaneously, assuming of course that inputs are mutually independent? Note that multiple-input multiple-output (MIMO) systems are typically identified as a series of multiple-input single-output systems.

In the literature, it is argued that in industry the guideline for identification practice is still single-variable based thinking [10]. However, several authors propose to excite all inputs simultaneously $[11,2]$. Still, there is no pertinent analysis of the variance to support these statements.

To obtain a reliable answer to the question stated in the first paragraph, we perform an analysis of the variance of the estimated parameters, in the parametric model structures most commonly used in system identification: FIR (Finite Impulse Response), ARX (Auto-Regressive with eXogeneous inputs), ARMAX (Auto-Regressive Moving-Average with eXogeneous inputs), OE (Output Error) and BJ (Box-Jenkins). In all cases, we assume that the system is in the model set. The expression for the distribution of parameter estimates that are asymptotic in the number of data is a classical result [6]. The theoretical conditions for the validity of this asymptotic theory have recently been treated in [3]. The question we address in our analysis of variance is: "What is the effect on the variance of the estimated coefficients of an additional input signal?". For example, consider an ARMAX model with two inputs: $A\left(z^{-1}\right) y(t)=B_{1}\left(z^{-1}\right) u_{1}(t)+B_{2}\left(z^{-1}\right) u_{2}(t)+C\left(z^{-1}\right) e(t)$ where $z^{-1}$ is the delay operator. What is the effect on the variance of the estimated coefficients of the $A, B_{1}$, and $C$ polynomials when the system is excited by two independent inputs $u_{1}$ and $u_{2}$, compared to the situation when $u_{2}=0$ ?

To motivate the reader, and to show that the answer to this question is not trivial, we take the rather unconventional approach of starting this paper with a quizz. We ask the reader to determine, for each of the following model structures, which of the polynomial estimates will be improved when the input signal $u_{2}$ is added, compared to the situation where only the signal $u_{1}$ is applied, i.e. $u_{2}=0$. The standing assumption is that the two input signals are independent, and that the true system is always in the model set. 


$$
\begin{array}{rlrl}
M_{1}: & A\left(z^{-1}\right) y(t) & =B_{1}\left(z^{-1}\right) u_{1}(t)+B_{2}\left(z^{-1}\right) u_{2}(t)+C\left(z^{-1}\right) e(t) \\
M_{2}: & B_{1} & =\frac{B_{1}\left(z^{-1}\right)}{F\left(z^{-1}\right)} u_{1}(t)+\frac{B_{2}\left(z^{-1}\right)}{F\left(z^{-1}\right)} u_{2}(t)+\frac{B_{1}\left(z^{-1}\right)}{D\left(z^{-1}\right)} e(t) \\
M_{3}: & & y(t) & =\frac{B\left(z^{-1}\right)}{F_{1}\left(z^{-1}\right)} u_{1}(t)+\frac{B\left(z^{-1}\right)}{F_{2}\left(z^{-1}\right)} u_{2}(t)+\frac{C\left(z^{-1}\right)}{D\left(z^{-1}\right)} e(t) \\
M_{4}: & & y(t) & =\frac{B_{1}\left(z^{-1}\right)}{F_{1}\left(z^{-1}\right)} u_{1}(t)+\frac{B_{2}\left(z^{-1}\right)}{D\left(z^{-1}\right)} u_{2}(t)+\frac{C\left(z^{-1}\right)}{D\left(z^{-1}\right)} e(t)
\end{array}
$$

The solution to the quizz is provided at the end of Section 4.

It quickly became clear to us that the effect of an additional input on the accuracy of the estimated polynomial coefficients, and the question of whether one should excite the inputs separately during part of the experimentation time, or rather simultaneously, might well be different for different model structures. The conclusion of our analysis is that, whatever the model structure, one never does worse with simultaneous excitation than with separate excitation and, for many model structures, one actually gets a smaller covariance matrix of the estimated polynomial coefficients, including coefficients related to the other input signals or to the noise model.

This result in itself is probably not too surprising, even though a number of system identification experts would probably be inclined to apply the excitation signals during separate experimentation periods in the case of a Multiple-Input Single-Output (MISO) system. However, our covariance analysis led to some results that run contrary to commonly held beliefs in the system identification community. Let us just foreshadow here two of these findings.

- It is a commonly held view that, in open-loop identification, the variance of the noise model is unaffected by the input signal. This view finds its origins in the widely used variance formula for the estimated noise model of a linear time-invariant system $y(t)=G(z) u(t)+H(z) e(t)$ :

$$
\operatorname{var}\left(\hat{H}_{N}\left(e^{j \omega}\right)\right) \simeq \frac{n}{N} \frac{\Phi_{v}\left(e^{j \omega}\right)}{\left|H\left(e^{j \omega}\right)\right|^{2}}
$$

in which the input signal plays no role whatsoever [6]. In this expression, $\Phi_{v}(\omega)$ is the power spectrum of the noise $v(t)=H(z) e(t)$ with $e(t)$ being a zero-mean white noise, $n$ is the order of the noise model, $N$ is the number of data, and $\hat{H}_{N}\left(e^{j \omega}\right)$ is the noise model estimate at frequency $\omega$. This formula, together with a corresponding formula for $\hat{G}_{N}\left(e^{j \omega}\right)$, was proposed by Ljung [5] under the assumption of model order and number of data tending both to infinity in some specific manner. Recently, exact variance expressions that are not asymptotic in model order have been derived in [9] and [7]. For example, Ninness and Hjalmarsson have considered the BJ and OE models and found that, in these cases, the variance of the noise model is 
independent of the input signal. However, no result is available for the ARX and ARMAX models, which are treated in this paper. We show in particular that, for the ARMAX model $A\left(z^{-1}\right) y(t)=B\left(z^{-1}\right) u(t)+C\left(z^{-1}\right) e(t)$ ( with $z^{-1}$ being the delay operator), the input signal $u(t)$ does in fact contribute to a reduction of the variance of $\hat{C}_{N}$, as well as that of $\hat{A}_{N}$.

- It follows from the first result that the addition of a second input signal, say $u_{2}(t)$, always reduces the variance of the estimated noise model $\frac{\hat{C}_{N}\left(e^{j \omega}\right)}{\hat{A}_{N}\left(e^{j \omega}\right)}$ of an ARMAX model. In addition, we show that, for ARX and ARMAX models, the addition of a signal $u_{2}(t)$ also improves the estimation of the polynomial $B_{1}\left(z^{-1}\right)$ related to the first input.

Our results do not contradict any established result in the identification literature. The longstanding variance formulas based on asymptotic model orders have contributed to the widely held but incorrect view that the input signal has no bearing on the precision of the noise model in open-loop identification. These formulas are presently being reassessed and replaced by more precise formulas for finite order models. The results of this paper are part of this reassessment.

Our most significant new contributions, in this respect, are our analysis of the role of the input signal in the increased precision of all parameters for ARMAX model structures, as well as the necessary and sufficient conditions on the parametric structure of multiple-input single-output (MISO) model structures under which the addition of a new input term improves the accuracy of polynomial estimates.

Our second contribution concerns input design in the case of systems with several inputs, or where several input signals can be applied. Our results provide an answer to the question of whether or not one should excite all inputs simultaneously. They also suggest that, if possible, it pays to add an input into the system for the sake of identifying model parameters whenever the corresponding input-output transfer function has common parameters with some other transfer function appearing in the model structure. For example, if the physics of the problem allow one to convert the ARMAX system $A y=B u+C e$ to the system $A y=B u+B_{2} u_{2}+C e$, then the addition of the signal $u_{2}$ improves the precision of all other estimated polynomials $A, B$ and $C$. Such thought would certainly not have occurred to the authors prior to this piece of research. We shall leave it to the reader to decide whether some of our findings are surprizing or not.

The paper is organized as follows. Relevant material on Prediction Error Identification is given in Section 2. In Section 3, we present the variance analysis for systems with one output and two inputs, successively for FIR, ARX, ARMAX and BJ models. These results are extended to arbitrary MISO model structures in Section 4. Section 5 focuses on input design problems for the case 
of MISO systems: we show in that section that one can never do better than simultaneous excitation of all inputs. Section 6 brings some final remarks.

\section{Preliminaries on Prediction Error Identification}

In this section, we consider the identification of an unknown linear timeinvariant "true" system with one output and two inputs (hereafter denoted a DISO system, for double-input single-output):

$$
\mathcal{S}: y(t)=G_{1}\left(z, \theta_{0}\right) u_{1}(t)+G_{2}\left(z, \theta_{0}\right) u_{2}(t)+H\left(z, \theta_{0}\right) e(t),
$$

where $G_{1}\left(z, \theta_{0}\right)$ and $G_{2}\left(z, \theta_{0}\right)$ are strictly causal rational transfer functions, $H\left(z, \theta_{0}\right)$ is the stable and inversely stable noise model driven by a white noise input $e$ with variance $\sigma_{e}^{2}, y$ is the measured output, $u_{1}$ and $u_{2}$ are two input signals. A special case of (2) is a single-input single-output (SISO) system, when $u_{2}=0$. We consider the identification of a model for $\mathcal{S}$ using a parametrized model set:

$$
\mathcal{M}=\left\{G_{1}(z, \theta), G_{2}(z, \theta), H(z, \theta), \theta \in D_{\theta} \subset \mathrm{R}^{k}\right\}
$$

where $G_{1}(z, \theta), G_{2}(z, \theta)$ and $H(z, \theta)$ are rational transfer functions, $\theta \in \mathrm{R}^{k}$ is a parameter vector, and $D_{\theta}$ is a subset of admissible values for $\theta$. The analysis of this paper will focus entirely on variance errors; thus we assume from now on that $\mathcal{S} \in \mathcal{M}$, i.e. $\theta_{0} \in D_{\theta}$. To every $\theta$ corresponds a one-step ahead predictor $\hat{y}(t \mid t-1, \theta)$, and hence a one-step ahead prediction error:

$$
\begin{aligned}
\varepsilon(t, \theta) & \triangleq y(t)-\hat{y}(t \mid t-1, \theta) \\
& =H^{-1}(z, \theta)\left[y(t)-G_{1}(z, \theta) u_{1}(t)-G_{2}(z, \theta) u_{2}(t)\right]
\end{aligned}
$$

The least-squares PE estimate $\hat{\theta}_{N}$ based on $N$ input-output data is defined as

$$
\hat{\theta}_{N}=\arg \min _{\theta \in D_{\theta}} V_{N}(\theta), \quad \text { with } V_{N}(\theta)=\frac{1}{N} \sum_{t=1}^{N}[\varepsilon(t, \theta)]^{2} .
$$

The estimate $\hat{\theta}_{N}$ defines the model $\hat{G}_{1}=G_{1}\left(z, \hat{\theta}_{N}\right), \hat{G}_{2}=G_{2}\left(z, \hat{\theta}_{N}\right), \hat{H}=$ $H\left(z, \hat{\theta}_{N}\right)$. Since $\mathcal{S} \in \mathcal{M}$, it follows that under reasonable conditions $\hat{\theta}_{N} \stackrel{N \rightarrow \infty}{\longrightarrow} \theta_{0}$. Thus, the transfer function estimates converge to the true transfer functions. The parameter error then converges to a Gaussian random variable [6]:

$$
\sqrt{N}\left(\hat{\theta}_{N}-\theta_{0}\right) \stackrel{\mathcal{D}}{\longrightarrow} \mathcal{N}\left(0, P_{\theta}\right), \quad \text { as } N \rightarrow \infty
$$

where

$$
P_{\theta}=\sigma_{e}^{2}\left[E \psi\left(t, \theta_{0}\right) \psi^{T}\left(t, \theta_{0}\right)\right]^{-1} \triangleq \sigma_{e}^{2} M^{-1}
$$


with an obvious definition for the information matrix $M \in \mathrm{R}^{k \times k}$. Here $\psi(t, \theta)$ is the gradient of the prediction error with respect to the parameters (i.e. the sensitivity of these errors to parameter variations):

$$
\psi(t, \theta) \triangleq \frac{\partial \varepsilon(t, \theta)}{\partial \theta}=-\frac{\partial \hat{y}(t \mid t-1, \theta)}{\partial \theta}
$$

It follows from the formulas (8), (4) and (2) that this gradient $\psi$, evaluated at $\theta=\theta_{0}$, can be rewritten as follows:

$$
\begin{aligned}
\psi\left(t, \theta_{0}\right)=\frac{-1}{H\left(z, \theta_{0}\right)} & {\left[\left.\frac{\partial G_{1}(z, \theta)}{\partial \theta}\right|_{\theta=\theta_{0}} u_{1}(t)\right.} \\
& \left.+\left.\frac{\partial G_{2}(z, \theta)}{\partial \theta}\right|_{\theta=\theta_{0}} u_{2}(t)+\left.\frac{\partial H(z, \theta)}{\partial \theta}\right|_{\theta=\theta_{0}} e(t)\right] .
\end{aligned}
$$

Combining (7) and (9), and using Parseval's theorem, the information matrix $M$ can then be written as:

$$
\begin{aligned}
M=\frac{1}{2 \pi} \int_{-\pi}^{\pi} \frac{1}{|H|^{2}}\left\{\left(\frac{\partial G_{1}}{\partial \theta}\right)\left(\frac{\partial G_{1}}{\partial \theta}\right)^{*} \Phi_{u_{1}}\right. \\
\left.+\left(\frac{\partial G_{2}}{\partial \theta}\right)\left(\frac{\partial G_{2}}{\partial \theta}\right)^{*} \Phi_{u_{2}}+\left(\frac{\partial H}{\partial \theta}\right)\left(\frac{\partial H}{\partial \theta}\right)^{*} \sigma_{e}^{2}\right\} d \omega
\end{aligned}
$$

where all quantities are evaluated at $\theta=\theta_{0}$. For a sufficiently large number $N$ of data, the asymptotic covariance formulas (6)-(7) are typically used to compute approximate expressions for the covariance of the parameter vector estimate:

$$
\operatorname{cov}\left(\hat{\theta}_{N}\right) \approx \frac{1}{N} P_{\theta}=\frac{\sigma_{e}^{2}}{N} M^{-1}
$$

Expression (10) will be our key tool for the analysis of the effect of one input signal, say $u_{2}$, on the covariance of the estimates of the various model polynomial coefficients. For example, by specializing this formula to an ARMAX model structure $A\left(z^{-1}\right) y(t)=B_{1}\left(z^{-1}\right) u_{1}(t)+B_{2}\left(z^{-1}\right) u_{2}(t)+C\left(z^{-1}\right) e(t)$, one can examine whether or not the input $u_{2}$ reduces the variance of some or all of the estimated coefficients of the polynomials $A, B_{1}$ and $C$. We mention that the gradients appearing in (10) have been computed in [4] for all commonly used polynomial model structures (FIR, ARX, OE, BJ, ARMAX).

For model structures that are linear in the parameters, such as FIR or ARX, the one-step ahead predictor can be written as:

$$
\hat{y}(t \mid t-1, \theta)=\varphi^{T}(t) \theta
$$

where $\varphi(t) \in \mathrm{R}^{k}$ is a vector containing known input and output signals only, i.e. independent of $\theta$. In such cases, the matrix $M=E \varphi(t) \varphi^{T}(t)$ is very 
easy to compute, and there is no need to resort to the frequency domain integral expression (10). Finally, we shall need the following technical result on persistently exciting signals.

Definition 1 [6],[8] Let $u(t)$ be a quasi-stationary signal, and denote $U^{T}(t-1) \triangleq[u(t-1) \ldots u(t-n)]$. Then $u(t)$ is called persistently exciting of order $n$ if and only if the matrix $R_{U U} \triangleq E\left\{U(t-1) U^{T}(t-1)\right\}$ is positive definite.

Proposition 1 [8] Let $y(t)$ be the output of an asymptotically stable rational filter $\frac{B\left(z^{-1}\right)}{A\left(z^{-1}\right)}$ driven by the input signal $u(t)$, with $B\left(z^{-1}\right)=\sum_{j=1}^{n_{b}} b^{j} z^{-j}$ and $A\left(z^{-1}\right)=1+\sum_{j=1}^{n_{a}} a^{j} z^{-j}$. Define the regression vector $\varphi^{T}(t)=[-y(t-1) \ldots-$ $\left.y\left(t-n_{a}\right) ; u(t-1) \ldots u\left(t-n_{b}\right)\right]$. Then, the covariance matrix $R \triangleq E\left\{\varphi(t) \varphi^{T}(t)\right\}$ is positive definite if and only if $u(t)$ is persistently exciting of order $n_{a}+n_{b}$ and the polynomials $A(z)$ and $B(z)$ are coprime.

Proof. See Complement C5.1 in [8].

In this paper we shall consider the identification of multiple-input rational systems of the following general form:

$$
y(t)=G_{1}(z) u_{1}(t)+G_{2}(z) u_{2}(t)+\cdots+G_{m}(z) u_{m}(t)+H(z) e(t),
$$

where the $G_{i}(z)$ are scalar strictly causal rational transfer functions, and $H(z)$ is a stable and inversely stable rational transfer function. Throughout the paper we shall make the following standing assumptions about the input signals $u_{1}, \ldots, u_{m}$.

\section{Standing Assumptions:}

(1) The inputs to the system (13) are quasi-stationary signals which are statistically independent from one another [6].

(2) Each input signal $u_{i}(t)$ is persistently exciting of order $n_{i}$, where $n_{i}$ is the sum of the degrees of the numerator and denominator polynomials of $G_{i}(z)$.

\section{Effect of a second input on classical polynomial model structures}

In this section, we use the formula (10), or its simplified version $M=$ $E \varphi(t) \varphi^{T}(t)$ whenever appropriate, to study the effect of the addition of a second input on the variance of the parameter estimates, for the classical FIR, ARX, ARMAX and BJ models. 


\subsection{FIR models}

Consider that the true system is described by the following FIR model structure:

$$
y(t)=B_{1}\left(z^{-1}\right) u_{1}(t)+B_{2}\left(z^{-1}\right) u_{2}(t)+e(t),
$$

where $B_{1}\left(z^{-1}\right)=\sum_{j=1}^{n} b_{1}^{j} z^{-j}$ and $B_{2}\left(z^{-1}\right)=\sum_{j=1}^{n} b_{2}^{j} z^{-j}$ are polynomials in the delay operator $z^{-1}$. For simplicity, we shall throughout this paper choose all polynomials in our models to have the same degree. The system can be rewritten in linear regression form:

$$
y(t)=\varphi^{T}(t) \theta_{0}+e(t)
$$

with

$$
\begin{aligned}
\theta_{0}^{T} & =\left[b_{1}^{1} \ldots b_{1}^{n} ; b_{2}^{1} \ldots b_{2}^{n}\right] \triangleq\left[\mathbf{b}_{1}^{T} ; \mathbf{b}_{2}^{T}\right] \\
\varphi^{T}(t) & =\left[u_{1}(t-1) \ldots u_{1}(t-n) ; u_{2}(t-1) \ldots u_{2}(t-n)\right] \\
& \triangleq\left[U_{1}^{T}(t-1) ; U_{2}^{T}(t-1)\right],
\end{aligned}
$$

with the obvious definitions for the $n$-dimensional vectors $\mathbf{b}_{\mathbf{1}}, \mathbf{b}_{\mathbf{2}}, U_{1}(t-1)$ and $U_{2}(t-1)$. The least-squares parameter estimate $\hat{\theta}_{N}$ of $\theta_{0}$ based on $N$ input-output data is unbiased, and its covariance, for large $N$, is given by (11) with

$$
P_{\theta}=\sigma_{e}^{2}\left(\begin{array}{cc}
R_{U_{1} U_{1}} & 0 \\
0 & R_{U_{2} U_{2}}
\end{array}\right)^{-1}
$$

where $R_{U_{i} U_{i}} \triangleq E\left\{U_{i}(t-1) U_{i}^{T}(t-1)\right\}$ for $i=1,2$. In the special case where the inputs are white noises with variance $\sigma_{u_{1}}^{2}$ and $\sigma_{u_{2}}^{2}$, respectively, we get the following approximate formula:

$$
\operatorname{cov}\left(\hat{\theta}_{N}\right) \approx \frac{1}{N}\left(\begin{array}{cc}
\frac{\sigma_{e}^{2}}{\sigma_{u_{1}}^{2}} I_{n} & 0 \\
0 & \frac{\sigma_{e}^{2}}{\sigma_{u_{2}}^{2}} I_{n}
\end{array}\right)
$$

\section{Analysis of the FIR case}

(1) The asymptotic accuracy of the estimates of the $b_{1}^{j}$ coefficients is independent of the presence of $u_{2}$. Thus, even if in the output signal $y$ the part due to the signal $u_{2}$ accounts for $90 \%$ of the total power, say, this has no effect on the quality of the estimates of the $B_{1}$ coefficients.

(2) For a white noise input signal $u_{1}$, the accuracy of the estimate of each coefficient $b_{1}^{j}$ is identical; it depends only on the signal to noise ratio $\frac{\sigma_{u_{1}}^{2}}{\sigma_{e}^{2}}$. 


\subsection{ARX models}

Consider now an ARX model structure with two independent inputs:

$$
A\left(z^{-1}\right) y(t)=B_{1}\left(z^{-1}\right) u_{1}(t)+B_{2}\left(z^{-1}\right) u_{2}(t)+e(t)
$$

where $A\left(z^{-1}\right)=1+\sum_{j=1}^{n} a^{j} z^{-j}$ and $B_{1}\left(z^{-1}\right), B_{2}\left(z^{-1}\right)$ are as before. The system can also be rewritten in the linear regression form (15), where $\phi(t)$ and $\theta_{0}$ are now defined as

$$
\begin{aligned}
\theta_{0}^{T} & =\left[a^{1} \ldots a^{n} ; b_{1}^{1} \ldots b_{1}^{n} ; b_{2}^{1} \ldots b_{2}^{n}\right] \triangleq\left[\mathbf{a}^{T} ; \mathbf{b}_{1}^{T} ; \mathbf{b}_{2}^{T}\right] \\
\varphi^{T}(t) & =\left[-y(t-1) \ldots-y(t-n) ; u_{1}(t-1) \ldots u_{1}(t-n) ; u_{2}(t-1) \ldots u_{2}(t-n)\right] \\
& \triangleq\left[Y^{T}(t-1) ; U_{1}^{T}(t-1) ; U_{2}^{T}(t-1)\right],
\end{aligned}
$$

with the obvious definitions for the $n$-dimensional vectors $\mathbf{a}, \mathbf{b}_{\mathbf{1}}, \mathbf{b}_{\mathbf{2}}, Y(t-1)$, $U_{1}(t-1)$ and $U_{2}(t-1)$. For an ARX model, the covariance, for large $N$, can be approximated by (11), where $M$ is now given by

$$
M=\left(\begin{array}{ccc}
R_{Y Y} & R_{Y U_{1}} & R_{Y U_{2}} \\
R_{U_{1} Y} & R_{U_{1} U_{1}} & 0 \\
R_{U_{2} Y} & 0 & R_{U_{2} U_{2}}
\end{array}\right)
$$

with $R_{Y Y} \triangleq E\left\{Y(t-1) Y^{T}(t-1)\right\}, R_{Y U_{1}} \triangleq E\left\{Y(t-1) U_{1}^{T}(t-1)\right\}$, etc. In order to apprehend the effect of the input signals on the accuracy of the parameter estimates of the polynomials $A, B_{1}$ and $B_{2}$, we need to compute, blockwise, the inverse of the information matrix $M$. We denote:

$$
C \triangleq M^{-1}=\left(\begin{array}{ccc}
C_{\mathbf{a} \mathbf{a}} & C_{\mathbf{a b}_{1}} & C_{\mathbf{a b}_{2}} \\
C_{\mathbf{b}_{1} \mathbf{a}} & C_{\mathbf{b}_{1} \mathbf{b}_{1}} & C_{\mathbf{b}_{1} \mathbf{b}_{2}} \\
C_{\mathbf{b}_{2} \mathbf{a}} & C_{\mathbf{b}_{2} \mathbf{b}_{1}} & C_{\mathbf{b}_{2} \mathbf{b}_{2}}
\end{array}\right)
$$

Remember that $P_{\theta}=\sigma_{e}^{2} M^{-1}=\sigma_{e}^{2} C$; in particular $\operatorname{cov}(\widehat{\mathbf{a}}) \approx \frac{\sigma_{e}^{2}}{N} C_{\mathbf{a a}}, \operatorname{cov}\left(\widehat{\mathbf{b}_{\mathbf{1}}}\right) \approx$ $\frac{\sigma_{e}^{2}}{N} C_{\mathbf{b}_{\mathbf{1}} \mathbf{b}_{\mathbf{1}}}$, and $\operatorname{cov}\left(\widehat{\mathbf{b}_{\mathbf{2}}}\right) \approx \frac{\sigma_{e}^{2}}{N} C_{\mathbf{b}_{\mathbf{2}} \mathbf{b}_{\mathbf{2}}}$.

We now compare the covariances of the estimates of the parameter vectors $\mathbf{a}$, $\mathbf{b}_{1}$ and $\mathbf{b}_{\mathbf{2}}$, based on $N$ input-output data, for the case where two independent input signals, $u_{1}$ and $u_{2}$, are applied simultaneously with the case where only one input is applied (i.e. $u_{2}=0$ ). We use the superscripts $(2)$ and (1) to distinguish between these two excitation conditions. For the case of two simultaneous and independent inputs, we have: 


$$
\begin{aligned}
C_{\mathbf{a a}}^{(2)} & =\left(R_{Y Y}-R_{Y U_{1}} R_{U_{1} U_{1}}^{-1} R_{U_{1} Y}-R_{Y U_{2}} R_{U_{2} U_{2}}^{-1} R_{U_{2} Y}\right)^{-1} \\
C_{\mathbf{b}_{1} \mathbf{b}_{1}}^{(2)} & =\left(R_{U_{1} U_{1}}-R_{U_{1} Y}\left(R_{Y Y}-R_{Y U_{2}} R_{U_{2} U_{2}}^{-1} R_{U_{2} Y}\right)^{-1} R_{Y U_{1}}\right)^{-1}
\end{aligned}
$$

When only $u_{1}$ is applied (i.e. $u_{2}=0$ ), the matrix $M$ reduces to its first two block rows and columns. For this case, the block-diagonal elements of its inverse matrix $C$ are:

$$
\begin{aligned}
C_{\mathbf{a a}}^{(1)} & =\left(R_{Y Y}-R_{Y U_{1}} R_{U_{1} U_{1}}^{-1} R_{U_{1} Y}\right)^{-1} \\
C_{\mathbf{b}_{\mathbf{1}} \mathbf{b}_{\mathbf{1}}}^{(1)} & =\left(R_{U_{1} U_{1}}-R_{U_{1} Y} R_{Y Y}^{-1} R_{Y U_{1}}\right)^{-1}
\end{aligned}
$$

We can now establish the following result.

Theorem 1 Consider the identification of the parameter vectors $\mathbf{a}$ and $\mathbf{b}_{\mathbf{1}}$ of the ARX model (18) under the Standing Assumptions. Then, the covariance matrices of the parameter estimates $\widehat{\mathbf{a}}$ and $\widehat{\mathbf{b}}_{\mathbf{1}}$ are smaller if $u_{2} \neq 0$ than if $u_{2}=0$, i.e.

$$
C_{\mathbf{a a}}^{(2)}<C_{\mathbf{a a}}^{(1)} \text { and } C_{\mathbf{b}_{\mathbf{1}} \mathbf{b}_{\mathbf{1}}}^{(2)}<C_{\mathbf{b}_{\mathbf{1}} \mathbf{b}_{\mathbf{1}}}^{(1)}
$$

Proof. In performing the comparison, we must keep in mind that the covariances $R_{Y Y}$ are different in these two situations. To take this into account, we split up the covariance matrix $R_{Y Y}$ into the sum of its contributions due to $u_{1}, u_{2}$ and $e$, respectively (see $(10)$ ):

$$
R_{Y Y}=R_{Y Y}\left(u_{1}\right)+R_{Y Y}\left(u_{2}\right)+R_{Y Y}(e) .
$$

By the Standing Assumption (2) and Proposition 1, it follows that

$$
\left(\begin{array}{cc}
R_{Y Y}\left(u_{2}\right) & R_{Y U_{2}} \\
R_{U_{2} Y} & R_{U_{2} U_{2}}
\end{array}\right)>0
$$

Comparing (21) with (22), we now observe that:

$$
\left(C_{\mathbf{a a}}^{(2)}\right)^{-1}-\left(C_{\mathbf{a a}}^{(1)}\right)^{-1}=R_{Y Y}\left(u_{2}\right)-R_{Y U_{2}} R_{U_{2} U_{2}}^{-1} R_{U_{2} Y}>0,
$$

where the last inequality follows from (24). Hence $C_{\mathbf{a a}}^{(2)}<C_{\mathbf{a a}}^{(1)}$. Comparing $C_{\mathbf{b}_{1} \mathbf{b}_{\mathbf{1}}}^{(1)}$ and $C_{\mathbf{b}_{1} \mathbf{b}_{\mathbf{1}}}^{(2)}$, we observe that they differ by the inverse that appears in the middle of their second term. In the case where $u_{2}=0$, the term to be inverted is $R_{Y Y}\left(u_{1}\right)+R_{Y Y}(e)$. When $u_{2} \neq 0$, that term becomes $R_{Y Y}\left(u_{1}\right)+$ $R_{Y Y}(e)+R_{Y Y}\left(u_{2}\right)-R_{Y U_{2}} R_{U_{2} U_{2}}^{-1} R_{U_{2} Y}$. For the same reason as above, the latter term is always strictly larger than the former, and hence $C_{\mathbf{b}_{\mathbf{1}} \mathbf{b}_{\mathbf{1}}}^{(2)}<C_{\mathbf{b}_{\mathbf{1}} \mathbf{b}_{\mathbf{1}}}^{(1)}$. 


\section{Analysis of the ARX case}

(1) Addition of a second input signal in an ARX system improves the accuracy of all parameter estimates. While the improved accuracy of the estimate of the $A$ polynomial is easily understood by the increased energy in the $y(t)$ data resulting in a better signal to noise ratio, the improvement of the estimate of the polynomial $B_{1}$ as a result of the addition of the input signal $u_{2}$ may come as a surprise. In fact, this improved accuracy results from the improved accuracy of the $A$ polynomial estimate and the correlation between the estimates of the $A$ and $B_{1}$ polynomial coefficients. Thus, the effect of the additional signal $u_{2}$ on the accuracy of $\widehat{\mathbf{a}}$ is direct, while the effect on $\widehat{\mathbf{b}_{\mathbf{1}}}$ is indirect via $\widehat{\mathbf{a}}$.

(2) To get a better understanding of this, we examine what happens when we let the energy of the signal $u_{2}$ increase. Consider that the formulas derived above have been written for some variance $\sigma_{u_{2}}^{2}$ of the signal $u_{2}$, and let us now replace this variance by $K \sigma_{u_{2}}^{2}$, with $K$ going to infinity. We examine the effect of $K$ on the covariances of $\widehat{\mathbf{a}}$ and $\widehat{\mathbf{b}_{\mathbf{1}}}$, respectively. Using (21) and (23), we get:

$$
\begin{aligned}
\left(C_{\mathbf{a a}}^{(2)}\right)^{-1}= & R_{Y Y}\left(u_{1}\right)+R_{Y Y}(e)-R_{Y U_{1}} R_{U_{1} U_{1}}^{-1} R_{U_{1} Y} \\
& +K\left[R_{Y Y}\left(u_{2}\right)-R_{Y U_{2}} R_{U_{2} U_{2}}^{-1} R_{U_{2} Y}\right] \\
\left(C_{\mathbf{b}_{\mathbf{1}} \mathbf{b}_{1}}^{(2)}\right)^{-1}= & R_{U_{1} U_{1}}-R_{U_{1} Y}\left[R_{Y Y}\left(u_{1}\right)+R_{Y Y}(e)\right. \\
& \left.+K\left(R_{Y Y}\left(u_{2}\right)-R_{Y U_{2}} R_{U_{2} U_{2}}^{-1} R_{U_{2} Y}\right)\right]^{-1} R_{Y U_{1}}
\end{aligned}
$$

It follows from these expressions that

$$
\lim _{K \rightarrow \infty}\left(C_{\mathbf{a a}}^{(2)}\right)^{-1}=\infty, \text { and } \lim _{K \rightarrow \infty}\left(C_{\mathbf{b}_{\mathbf{1}} \mathbf{b}_{\mathbf{1}}}^{(2)}\right)^{-1}=R_{U_{1} U_{1}} .
$$

Thus, when the energy of the additional input $u_{2}$ grows indefinitely, the covariance of $\widehat{\mathbf{a}}$ goes to zero, while that of $\widehat{\mathbf{b}_{\mathbf{1}}}$ goes to the covariance that would be obtained for a FIR model with input $u_{1}$ only. The intuition behind this last observation is that, when the variance of $u_{2}$ goes to infinity, the parameters of the $A$ and $B_{2}$ polynomials become perfectly known, and the estimation of the $B_{1}$ polynomial reduces to that of a FIR model $y(t)=B_{1}\left(z^{-1}\right) u_{1}(t)+e(t)$.

\section{Simulation example}

We consider the DISO ARX model structure (18) with $A=1-0.2 z^{-1}$, $B_{1}=10 z^{-1}+z^{-2}, B_{2}=0.1 z^{-1}+4 z^{-2}$, where $u_{1}, u_{2}$, and $e$ are three independent white noises, with standard deviations $\sigma_{u_{1}}=2, \sigma_{u_{2}}=10$, and $\sigma_{e}=4$, respectively. We compare the situation where only input $u_{1}$ is applied with the situation where the two inputs are applied simultaneously. For each case, we compute the theoretical covariance matrix and also perform a Monte-Carlo simulation to confirm these theoretical results. 


\section{Case A: 1 input}

The theoretical asymptotic covariance matrix $P_{\theta}$ for $\theta=\left(a^{1}, b_{1}^{1}, b_{1}^{2}\right)$ is:

$$
P_{\theta}=\left(\begin{array}{ccc}
0.2954 & 0 & -2.9538 \\
0 & 4.0000 & 0 \\
-2.9538 & 0 & 33.5385
\end{array}\right)
$$

The variances of the 3 parameter estimates computed by 1000 Monte-Carlo runs are:

$$
0.2849 \quad 4.0171 \quad 32.7052
$$

\section{Case B: 2 inputs}

The theoretical covariance matrix for the vector $\theta=\left(a^{1}, b_{1}^{1}, b_{1}^{2}, b_{2}^{1}, b_{2}^{2}\right)$ is:

$$
P_{\theta}=\left(\begin{array}{ccccc}
0.0092 & 0 & -0.0921 & 0 & -0.0009 \\
0 & 4.0000 & 0 & 0 & 0 \\
-0.0921 & 0 & 4.9208 & 0 & 0.0092 \\
0 & 0 & 0 & 0.1600 & 0 \\
-0.0009 & 0 & 0.0092 & 0 & 0.1601
\end{array}\right)
$$

The variances obtained by Monte-Carlo runs are now:

$$
\begin{array}{lllll}
0.0088 & 4.0185 & 5.1151 & 0.1616 & 0.1580
\end{array}
$$

Comparing the two situations, we observe that the presence of the second input $u_{2}$ not only reduces the variance of $a^{1}$ drastically, but it also significantly improves the precision of the estimated coefficient $b_{1}^{2}$. Note that it has no effect on $b_{1}^{1}$ because of the sampling delay. The Monte-Carlo simulations thus confirm the theoretical calculations. With the same Monte-Carlo runs, we also compute the experimental variances of the transfer function estimates $\frac{\widehat{B}_{1}}{\widehat{A}}$, evaluated at 500 frequency points. The results are shown in Figure 1: full line for the 2input system, dashed line for the one-input case. The absolute values of the variances are represented.

\subsection{ARMAX models}

For an ARMAX system, we need to refer to the frequency domain expression (10), with the general expressions of the sensitivities specialized to the ARMAX case. As we shall see, the effect of adding a second input signal in an ARMAX model structure follows immediately from the covariance analysis of 


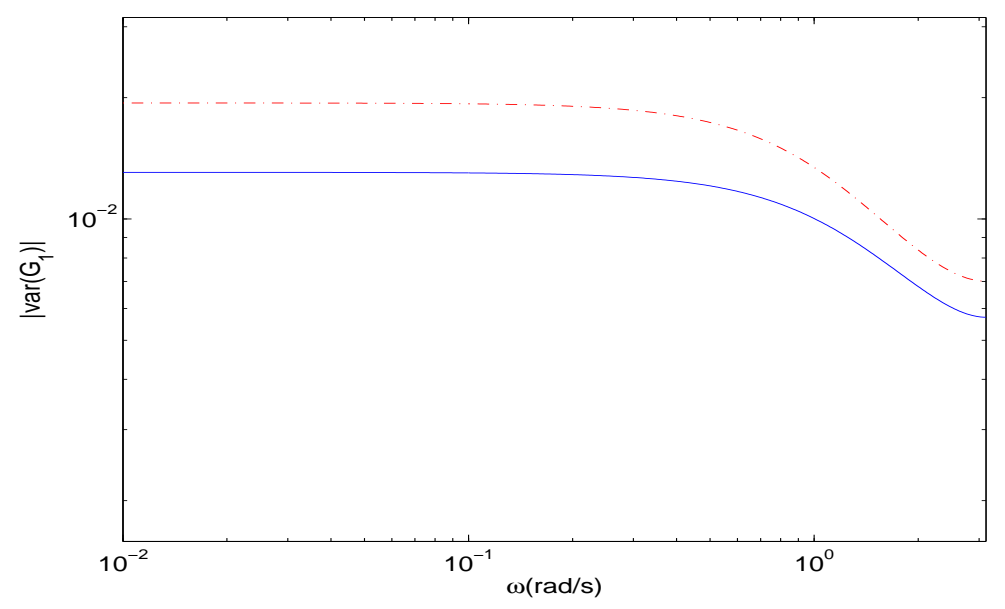

Fig. 1. Variance of $\frac{\widehat{B}_{1}}{\widehat{A}}$ for the ARX model with 2 inputs (full line) and one input (dashed line).

a SISO ARMAX model structure. Thus, we first consider the SISO ARMAX structure:

$$
A\left(z^{-1}\right) y(t)=B\left(z^{-1}\right) u(t)+C\left(z^{-1}\right) e(t)
$$

with $A$ and $B$ as before and $C\left(z^{-1}\right)=1+\sum_{j=1}^{n} c^{j} z^{-j}$. The parameter vector is defined as:

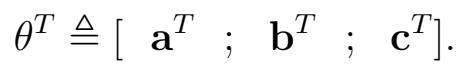

For this ARMAX model, the two terms in the expression (10) of the information matrix become:

$$
\frac{1}{H} \frac{\partial G}{\partial \theta}=\frac{1}{C}\left(\begin{array}{c}
-\frac{B}{A} e^{-j \omega} \\
\vdots \\
\frac{-\frac{B}{A} e^{-j \omega n}}{e^{-j \omega}} \\
\vdots \\
\frac{e^{-j \omega n}}{0} \\
\vdots \\
0
\end{array}\right), \frac{1}{H} \frac{\partial H}{\partial \theta}=\frac{1}{C}\left(\begin{array}{c}
-\frac{C}{A} e^{-j \omega} \\
\vdots \\
\frac{-\frac{C}{A} e^{-j \omega n}}{0} \\
\vdots \\
0 \\
e^{-j \omega} \\
\vdots \\
e^{-j \omega n}
\end{array}\right)
$$

With these expressions under our belt, we observe that the information matrix $M$ for an ARMAX model takes the following block-diagonal structure:

$$
M=\left(\begin{array}{ccc}
M_{\mathbf{a a}}(u)+M_{\mathbf{a a}}(e) & M_{\mathbf{a b}}(u) & M_{\mathbf{a c}}(e) \\
M_{\mathbf{b a}}(u) & M_{\mathbf{b b}}(u) & 0 \\
M_{\mathbf{c a}}(e) & 0 & M_{\mathbf{c c}}(e)
\end{array}\right)
$$


where, e.g., $M_{\mathrm{aa}}(u)$ denotes the contribution of the $(1,1)$-term of the information matrix that is due to the signal $u$. By computing the inverse $C=M^{-1}$ of (28), we can then study the effect of the input signal $u$ on the precision of each of the components $\hat{\mathbf{a}}, \hat{\mathbf{b}}$, and $\hat{\mathbf{c}}$ of the parameter estimate $\hat{\theta}$. We have the following result.

Theorem 2 Consider the identification of the parameters of the ARMAX model (27) under the Standing Assumptions. The input signal u contributes to the estimation of all parameter estimates, $\hat{\mathbf{a}}, \hat{\mathbf{b}}$ and $\hat{\mathbf{c}}$. In particular, the covariance matrix of $\hat{\mathbf{c}}$ has the following expression:

$$
\begin{aligned}
P_{\mathbf{c}}(A R M A X)=\sigma_{e}^{2}\left\{M_{\mathbf{c c}}(e)\right. & -M_{\mathbf{c a}}(e)\left[M_{\mathbf{a a}}(u)+M_{\mathbf{a a}}(e)\right. \\
& \left.\left.-M_{\mathbf{a b}}(u) M_{\mathbf{b b}}^{-1}(u) M_{\mathbf{b a}}(u)\right]^{-1} M_{\mathbf{a c}}(e)\right\}^{-1}
\end{aligned}
$$

For the corresponding ARMA model structure (i.e. no input) with the same A and $C$ polynomial, the covariance of the estimate of the $C$ polynomial is larger than in the ARMAX model structure:

$$
P_{\mathbf{c}}(A R M A)=\sigma_{e}^{2}\left\{M_{\mathbf{c c}}(e)-M_{\mathbf{c a}}(e) M_{\mathbf{a a}}^{-1}(e) M_{\mathbf{a c}}(e)\right\}^{-1}>P_{\mathbf{c}}(A R M A X)
$$

Proof. Expression (29) follows by straightforward calculation of the $(3,3)$ block-element of $M^{-1}$, while (30) is obtained from the corresponding formulas for the ARMA model case. By comparing expressions (29) and (30), and noting that $M_{\mathbf{a a}}(u)-M_{\mathbf{a b}}(u) M_{\mathbf{b b}}^{-1}(u) M_{21}(u)>0$ by Proposition 1, it follows immediately that $P_{\mathbf{c}}(A R M A)>P_{\mathbf{c}}(A R M A X)$.

Corollary 1 Consider the identification of the parameter vectors $\mathbf{a}, \mathbf{b}_{1}$ and $\mathbf{c}$ of the ARMAX model:

$$
A\left(z^{-1}\right) y(t)=B_{1}\left(z^{-1}\right) u_{1}(t)+B_{2}\left(z^{-1}\right) u_{2}(t)+C\left(z^{-1}\right) e(t)
$$

under the Standing Assumptions on $u_{1}$ and $u_{2}$. Then, the covariance matrices of the parameter estimates $\widehat{\mathbf{a}}, \widehat{\mathbf{b}_{1}}$ and $\widehat{\mathbf{c}}$ are smaller if $u_{2} \neq 0$ than if $u_{2}=0$.

Proof. Theorem 1 states that $u_{2}$ reduces the covariance of $\widehat{\mathbf{a}}$ and $\widehat{\mathbf{b}_{1}}$, while Theorem 2 states that it also reduces the covariance of $\widehat{\mathbf{c}}$.

\section{Analysis of the ARMAX case}

(1) In an ARMAX system, the input signal $u$ affects the variance of the estimates of the $A, B$ and $C$ polynomials. In particular, the variance of the estimates of the $A$ and $C$ polynomials is smaller than in the corresponding ARMA model. 
(2) The addition of a second input in an ARMAX model structure reduces the covariance of the estimates of all polynomials.

(3) If the variance of the input signal $u$ grows indefinitely, expression (29) shows that the covariance matrix $P_{c}(A R M A X)$ for the parameter estimate $\hat{\mathbf{c}}$ tends to $\sigma_{e}^{2} M_{\mathbf{c c}}^{-1}(e)$. This is the variance that would be obtained for the identification of the corresponding Moving Average (MA) model $y(t)=C\left(z^{-1}\right) e(t)$. It is smaller than the corresponding $P_{c}(A R M A)$ of (30). The intuition, as for the similar observation made for the ARX model with two input signals, is that the polynomials $A$ and $B$ become perfectly known when the input signal power tends to infinity.

\section{Simulation example}

We have simulated the ARMAX model (27) with $A=1-0.2 z^{-1}$, $B=10 z^{-1}+z^{-2}$ and $C=1-1.6 z^{-1}+0.64 z^{-2}$, and with $u$ and $e$ two independent white noises with $\sigma_{u}=1$ and $\sigma_{e}=4$, respectively. We have compared, both theoretically and by Monte-Carlo simulations with 1000 runs, the covariances obtained for the parameter estimates for this ARMAX model with those obtained with the ARMA model with the same $A$ and $C$ polynomials, i.e. with $u=0$ in the above model.

\section{ARMA model}

The theoretical asymptotic covariance matrix $P_{\theta}$ with $\theta=\left(a^{1}, c^{1}, c^{2}\right)$ is:

$$
P_{\theta}=\left(\begin{array}{ccc}
3.6879 & 2.4837 & -2.3934 \\
2.4837 & 2.2631 & -2.1879 \\
-2.3934 & -2.1879 & 2.1437
\end{array}\right)
$$

The variances obtained by Monte-Carlo runs are:

$$
3.9433 \quad 2.4015 \quad 2.2896
$$

\section{ARMAX model}

The theoretical covariance matrix for the vector $\theta=\left(a^{1}, b^{1}, b^{2}, c^{1}, c^{2}\right)$ is:

$$
P_{\theta}=\left(\begin{array}{ccccc}
0.6906 & -1.8792 & 11.2279 & 0.4651 & -0.4482 \\
-1.8792 & 14.5597 & -39.7682 & -1.2656 & 1.2195 \\
11.2279 & -39.7682 & 191.9959 & 7.5617 & -7.2867 \\
0.4651 & -1.2656 & 7.5617 & 0.9036 & -0.8778 \\
-0.4482 & 1.2195 & -7.2867 & -0.8778 & 0.8813
\end{array}\right)
$$


The variances obtained with 1000 Monte-Carlo runs are:

\section{$\begin{array}{lllll}0.6805 & 15.8600 & 194.6594 & 0.9922 & 0.9735\end{array}$}

The Monte-Carlo simulations confirm the theoretical computations. We observe that the presence of an input signal in the ARMAX model, in comparison with the ARMA model, not only reduces the variance of the $a$ parameter (by a factor of 5 ), but also that of the $c^{1}$ and $c^{2}$ parameter estimates (by a factor of 2.5). We have also computed the variance of the estimated models $\frac{\widehat{C}}{\widehat{A}}$ obtained with the Monte-Carlo simulations for the ARMA and ARMAX models.

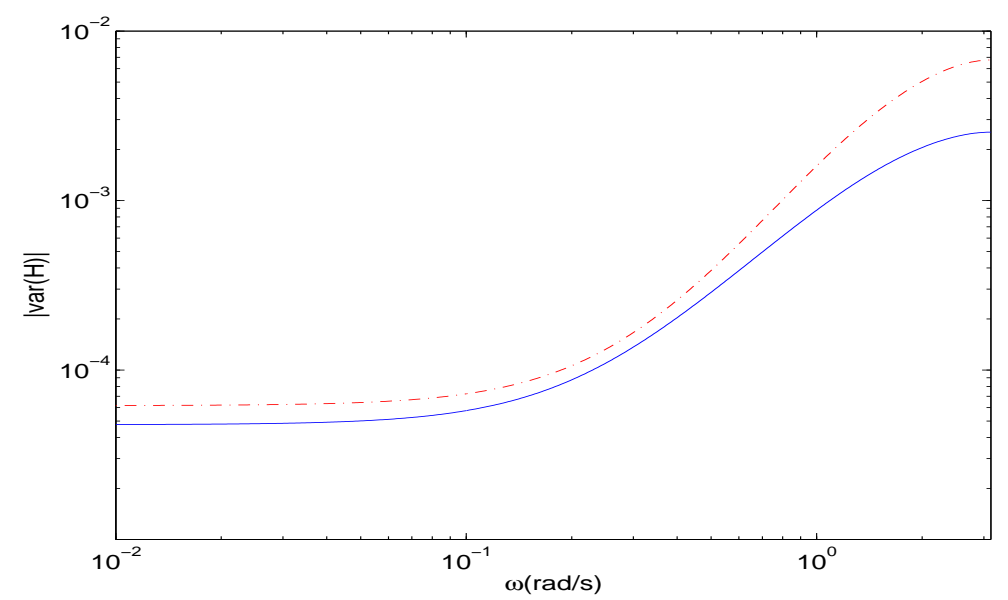

Fig. 2. Variance of $\frac{\widehat{C}}{\widehat{A}}$ for the ARMA (dashed line) and ARMAX (full line) models.

\section{$3.4 \quad$ Box-Jenkins models}

Consider now a BJ model structure with two independent inputs:

$$
y(t)=\frac{B_{1}\left(z^{-1}\right)}{F_{1}\left(z^{-1}\right)} u_{1}(t)+\frac{B_{2}\left(z^{-1}\right)}{F_{2}\left(z^{-1}\right)} u_{2}(t)+\frac{C\left(z^{-1}\right)}{D\left(z^{-1}\right)} e(t)
$$

where the polynomials $B_{1}, B_{2}$ and $C$ are as before, and the polynomials $F_{1}$, $F_{2}$ and $D$ are all monic of degree $n$. The parameter vector is defined as

$$
\theta^{T} \triangleq\left[\mathbf{b}_{1}^{T} ; \mathbf{f}_{1}^{T} ; \mathbf{b}_{2}^{T} ; \mathbf{f}_{2}^{T} ; \mathbf{c}^{T} ; \mathbf{d}^{T}\right]
$$


For a BJ model with a single input, the terms in the expression (10) of the information matrix specialize to:

$$
\frac{1}{H} \frac{\partial G}{\partial \theta}=\frac{D}{C F}\left(\begin{array}{c}
e^{-j \omega} \\
\vdots \\
\frac{e^{-j \omega n}}{-\frac{B}{F} e^{-j \omega}} \\
\vdots \\
-\frac{B}{F} e^{-j \omega n} \\
0 \\
\vdots \\
0 \\
0 \\
\vdots \\
0
\end{array}\right), \frac{1}{H} \frac{\partial H}{\partial \theta}=\left(\begin{array}{c}
0 \\
\vdots \\
0 \\
\hline 0 \\
\vdots \\
0 \\
-\frac{1}{C} e^{-j \omega} \\
\vdots \\
-\frac{1}{C} e^{-j \omega n} \\
-\frac{1}{D} e^{-j \omega} \\
\vdots \\
-\frac{1}{D} e^{-j \omega n}
\end{array}\right)
$$

Introducing these expressions into (10), we observe that the information matrix for the SISO BJ model structure is block-diagonal. With the addition of a second input signal, this information matrix takes the following form:

$$
M=\left(\begin{array}{ccc}
M_{11}\left(u_{1}\right) & 0 & 0 \\
0 & M_{22}\left(u_{2}\right) & 0 \\
0 & 0 & M_{33}(e)
\end{array}\right)
$$

\section{Analysis of the BJ case}

(1) With a BJ model structure, the input signal has no effect on the precision of the noise model estimate. This observation is valid for open-loop identification only. In closed-loop identification of BJ models, the input signal $u$ does influence the noise model estimate (see [1]).

(2) The effect of each input signal on the parameter estimate covariance is completely decoupled from the other input signals. Addition of a second input signal $u_{2}$ therefore does not improve the quality of the estimates of any polynomials not related to that input.

(3) The situation for OE models is identical to that for BJ models. 


\section{Generalization to arbitrary multi-input model structures}

In this section, we generalize the results of the previous section to arbitrary multi-input systems. We shall derive exact conditions on the structure of the parametrization of multi-input models under which the addition of a new input term improves the quality of the parameter estimates appearing in the other transfer functions. For the $m$-input 1-output system (13), the following completely general polynomial model structure can be written:

$$
y(t)=\frac{B_{1}\left(z^{-1}\right)}{F_{1}\left(z^{-1}\right)} u_{1}(t)+\frac{B_{2}\left(z^{-1}\right)}{F_{2}\left(z^{-1}\right)} u_{2}(t)+\cdots+\frac{B_{m}\left(z^{-1}\right)}{F_{m}\left(z^{-1}\right)} u_{m}(t)+\frac{C\left(z^{-1}\right)}{D\left(z^{-1}\right)} e(t) .
$$

Here $B_{i}, F_{i}, i=1, \ldots, m$, as well as $C$ and $D$ are polynomials in the shift operator $z^{-1}$, with $C$ and $D$ monic. These polynomials may or may not contain common parameters. For example, the ARMAX model with two inputs is a special case of (34) in which $m=2$, with $F_{1}=F_{2}=D=A$. As before, it is assumed that the true $m$-input 1-output system can be represented by (34) for a particular value of the parameters.

Consider first the estimates of the parameters of the model structure (34) obtained from an identification experiment $X_{1}$ with $m$ inputs for which the Standing Assumptions hold. Consider next a second identification experiment $X_{2}$, in which the input signals $u_{1}, \ldots, u_{m}$ are the same as before but with the application of an additional input term $\frac{B_{m+1}\left(z^{-1}\right)}{F_{m+1}\left(z^{-1}\right)} u_{m+1}(t)$, also obeying the Standing Assumptions. By the analysis of Section 3, we already know that if $B_{m+1}$ or $F_{m+1}$ has common parameters with some of the other transfer functions, this additional input will improve the quality of the estimates of these other transfer functions. However, the analysis of Section 3 does not tell us exactly which polynomial estimates are improved. One might be tempted to believe that the polynomial estimates that are improved by the addition of $\frac{B_{m+1}\left(z^{-1}\right)}{F_{m+1}\left(z^{-1}\right)} u_{m+1}(t)$ are those that appear in transfer functions that have common parameters with either $B_{m+1}$ or $F_{m+1}$. For example, on the basis of the analysis of Section 3, addition of the input term $\frac{B_{m+1}\left(z^{-1}\right)}{A\left(z^{-1}\right)} u_{m+1}(t)$ in an ARMAX system is expected to improve all polynomial estimates of the model, because the polynomial $A$ appears in all transfer functions. As our analysis will show, even though having parameters in common with the polynomials $B_{m+1}$ or $F_{m+1}$ appearing in the added input term is a sufficient condition for accuracy improvement, it is not a necessary condition. Thus, the quality improvement carries to more transfer function estimates than those that have common parameters with the added transfer function $\frac{B_{m+1}\left(z^{-1}\right)}{F_{m+1}\left(z^{-1}\right)}$.

Our procedure for deriving necessary and sufficient conditions for accuracy improvement is to first compute the covariance of the polynomial estimates of any one of the transfer functions $\frac{B_{i}\left(z^{-1}\right)}{F_{i}\left(z^{-1}\right)}, i=1, \ldots, m$ or $\frac{C\left(z^{-1}\right)}{D\left(z^{-1}\right)}$ of the system 
(34) when it is excited by $m$ inputs, and to then examine under what structural conditions the addition of the input term $\frac{B_{m+1}\left(z^{-1}\right)}{F_{m+1}\left(z^{-1}\right)} u_{m+1}(t)$ improves the quality of these parameter estimates when all other conditions are left unchanged and the Standing Assumptions hold.

In analyzing the effect of adding an extra input signal on the estimates of the polynomials appearing in other transfer functions, we first observe that no distinction is made between the covariance of the parameter estimates of the transfer functions $G_{i}(z)$ excited by the measured signals $u_{i}(t)$ and the covariance of the parameter estimates of the transfer function $H(z)$ excited by the unmeasured white noise signal $e(t)$ (see (9) and (10)). In other words, the conditions on the parametric structure under which the addition of an input term $\frac{B_{m+1}\left(z^{-1}\right)}{F_{m+1}\left(z^{-1}\right)} u_{m+1}(t)$ improves the estimates of the parameters of $B_{i}, F_{i}$ are identical to the conditions under which such additional input term improves the estimates of the parameters of the $C$ and $D$ polynomials in the model of $H(z)$. Since all considered models contain a noise term $H(z) e(t)$, and in order to keep the analysis as simple as possible, we thus seek necessary and sufficient conditions under which the parameter estimates of the noise model $H(z)=$ $\frac{C\left(z^{-1}\right)}{D\left(z^{-1}\right)}$ are improved by addition of an input term. The first lemma provides the conditions under which addition of the input term $G(z) u(t)$ improves the parameter estimates of the noise model.

In a first experiment, denoted $X_{1}$, we consider the identification of the system $\mathcal{S}_{1}: y(t)=H\left(z, \beta_{0}, \gamma_{0}\right) e(t)$ with a parametric model structure $\mathcal{M}_{1}: y(t)=$ $H(z, \beta, \gamma) e(t)$ using $N$ output data, where $\beta$ and $\gamma$ are parameter vectors. We compare the covariance of the parameter estimates $\hat{\beta}_{N}^{(1)}$ and $\hat{\gamma}_{N}^{(1)}$ obtained in experiment $X_{1}$ with the covariance of the estimates of these same parameter vectors obtained from $N$ input-output data collected on the following two systems:

$$
\begin{aligned}
& \mathcal{S}_{2}: y(t)=G\left(z, \alpha_{0}\right) u(t)+H\left(z, \beta_{0}, \gamma_{0}\right) e(t) \\
& \mathcal{S}_{3}: y(t)=G\left(z, \alpha_{0}, \beta_{0}\right) u(t)+H\left(z, \beta_{0}, \gamma_{0}\right) e(t)
\end{aligned}
$$

Here $u$ is a measured input signal, independent of the white noise signal $e$. In each case, we take a model structure that is able to represent the true system:

$$
\begin{aligned}
& \mathcal{M}_{2}: y(t)=G(z, \alpha) u(t)+H(z, \beta, \gamma) e(t) \\
& \mathcal{M}_{3}: y(t)=G(z, \alpha, \beta) u(t)+H(z, \beta, \gamma) e(t)
\end{aligned}
$$

Note that in the system $\mathcal{S}_{3}$ and the corresponding model structure $\mathcal{M}_{3}$, the added input term $G(z, \alpha, \beta) u(t)$ has common parameters with the noise model $H(z, \beta, \gamma)$, while in the system $\mathcal{S}_{2}$ the parameters of these two transfer functions are disjoint. 
Lemma 1 Consider the estimation of the parameter vector $\left(\beta^{T}, \gamma^{T}\right)$ of the noise model $H(z, \beta, \gamma)$ based on $N$ data under three different experimental conditions, representing three different combinations of data generation mechanism and model structure: $X_{1}=\left\{\mathcal{S}_{1} \in \mathcal{M}_{1}\right\}, X_{2}=\left\{\mathcal{S}_{2} \in \mathcal{M}_{2}\right\}, X_{3}=$ $\left\{\mathcal{S}_{3} \in \mathcal{M}_{3}\right\}$. Assume also that the Standing Assumptions on $u$ hold for experiments $X_{2}$ and $X_{3}$. Then, the covariance of the parameter vector estimate $\left(\hat{\beta}_{N}^{T}, \hat{\gamma}_{N}^{T}\right)$ is identical in experiments $X_{1}$ and $X_{2}$, while it is smaller in experiment $\mathcal{X}_{3}$ than in the other two experiments. The accuracy of both $\hat{\beta}_{N}$ and $\hat{\gamma}_{N}$ are improved by the presence of common parameters, and not just that of the common parameter vector $\hat{\beta}_{N}$.

Proof. For experiment $X_{1}$, the covariance matrix of the parameter vector estimate is

$$
\operatorname{cov}\left(\begin{array}{c}
\hat{\beta}_{N}^{(1)} \\
\hat{\gamma}_{N}^{(1)}
\end{array}\right)=\frac{\sigma_{e}^{2}}{N}\left(\begin{array}{cc}
M_{\beta \beta}(e) & M_{\beta \gamma}(e) \\
M_{\gamma \beta}(e) & M_{\gamma \gamma}(e)
\end{array}\right)^{-1} \triangleq \frac{\sigma_{e}^{2}}{N}\left(M^{(1)}\right)^{-1}
$$

where the structure of the information matrix $M^{(1)}$ follows from (10), with $M_{\beta \gamma}(e) \triangleq \frac{1}{2 \pi} \int_{-\pi}^{\pi} \frac{\sigma_{e}^{2}}{|H|^{2}}\left(\frac{\partial H}{\partial \beta}\right)\left(\frac{\partial H}{\partial \gamma}\right)^{*} d \omega$, and similarly for the other elements.

For experiment $X_{2}$, the information matrix for the estimation of the parameter vector $\left(\begin{array}{lll}\alpha^{T} & \beta^{T} & \gamma^{T}\end{array}\right)$ is block-diagonal with $M_{\alpha \alpha}(u)$ as the $(1,1)$ block-element and $M^{(1)}$ as the $(2,2)$ block-element. Therefore, the covariance of the estimate $\left(\begin{array}{ll}\hat{\beta}_{N}^{T} & \hat{\gamma}_{N}^{T}\end{array}\right)$ is again given by the right hand side of (39) for this experimental situation.

For experiment $X_{3}$, we get

$$
\operatorname{cov}\left(\begin{array}{c}
\hat{\alpha}_{N}^{(3)} \\
\hat{\beta}_{N}^{(3)} \\
\hat{\gamma}_{N}^{(3)}
\end{array}\right)=\frac{\sigma_{e}^{2}}{N}\left(\begin{array}{ccc}
M_{\alpha \alpha}(u) & M_{\alpha \beta}(u) & 0 \\
M_{\beta \alpha}(u) & M_{\beta \beta}(u)+M_{\beta \beta}(e) & M_{\beta \gamma}(e) \\
0 & M_{\gamma \beta}(e) & M_{\gamma \gamma}(e)
\end{array}\right)^{-1}
$$

It then follows that, for this experimental condition,

$$
\operatorname{cov}\left(\begin{array}{c}
\hat{\beta}_{N}^{(3)} \\
\hat{\gamma}_{N}^{(3)}
\end{array}\right)=\frac{\sigma_{e}^{2}}{N}\left(\begin{array}{cc}
M_{\beta \beta}(e)+M_{\beta \beta}(u)-M_{\beta \alpha}(u) M_{\alpha \alpha}^{-1}(u) M_{\alpha \beta}(u) & M_{\beta \gamma}(e) \\
M_{\gamma \beta}(e) & M_{\gamma \gamma}(e)
\end{array}\right)^{-1}
$$

By the Standing Assumption on $G(z, \alpha, \beta) u(t)$, the term $M_{\beta \beta}(u)-$ $M_{\beta \alpha}(u) M_{\alpha \alpha}^{-1}(u) M_{\alpha \beta}(u)$ is positive definite. Comparing (41) with (39), we conclude that the covariance of the estimate of $\left(\beta^{T} \gamma^{T}\right)$ is smaller with experiment $X_{3}$ than with the other two experiments. Not only is the covariance of the common parameter vector $\hat{\beta}_{N}$ made smaller by the addition of the input term 
$G\left(z, \alpha_{0}, \beta_{0}\right) u(t)$, but also that of the parameter vector $\hat{\gamma}_{N}$ that appears only in the noise model. With experimental conditions $X_{1}$ and $X_{2}$, we get

$$
\operatorname{cov}\left(\hat{\gamma}_{N}\right)=\frac{\sigma_{e}^{2}}{N}\left[M_{\gamma \gamma}(e)-M_{\gamma \beta}(e) M_{\beta \beta}^{-1}(e) M_{\beta \gamma}(e)\right]^{-1}
$$

while with experimental conditions $\mathcal{X}_{3}$ we get the strictly smaller value

$$
\begin{aligned}
\operatorname{cov}\left(\hat{\gamma}_{N}^{(3)}\right)=\frac{\sigma_{e}^{2}}{N}\left[M_{\gamma \gamma}(e)-\right. & M_{\gamma \beta}(e)\left(M_{\beta \beta}(e)+M_{\beta \beta}(u)\right. \\
& \left.\left.\quad-M_{\beta \alpha}(u) M_{\alpha \alpha}^{-1}(u) M_{\alpha \beta}(u)\right)^{-1} M_{\beta \gamma}(e)\right]^{-1}
\end{aligned}
$$

\section{Comments}

- The analysis made in Lemma 1 would be identical if $\mathcal{S}_{1}$ was replaced by $\tilde{\mathcal{S}}_{1}: y(t)=G_{1}\left(z, \beta_{0}, \gamma_{0}\right) u_{1}(t)+e(t)$, and if we were to study the effect of adding an input term $G_{2}\left(z, \alpha_{0}\right) u_{2}(t)$ (respectively $G_{2}\left(z, \alpha_{0}, \beta_{0}\right) u_{2}(t)$ ) on the accuracy of the parameter estimates of the model $G_{1}(z, \beta, \gamma)$.

- Lemma 1 shows that addition of the input term $G(z) u(t)$ increases the precision of all parameters that appear in the other transfer function if and only if the model $G(z, \theta)$ for this input term has common parameters with that other transfer function.

The next lemma shows that this increased accuracy extends to the parameters of all other transfer function models that have common parameters with the transfer function models whose accuracy has been increased as a result of the added input term, and not just to transfer function models that have common parameters with this added input term; in other words, the increased accuracy has a "snowball effect".

Lemma 2 Consider the estimation of the parameter vectors $\beta$ and $\gamma$ appearing jointly in a particular transfer function model $G_{i}(z, \beta, \gamma)$ or $H(z, \beta, \gamma)$ of the MISO model (34). Consider two different experimental conditions, $X_{1}$ and $X_{2}\left(X_{2}\right.$ is the same as $X_{1}$ but with an additional input term), and assume that the covariance of $\hat{\beta}_{N}$ is smaller in experiment $X_{2}$ than in experiment $X_{1}$ : $\operatorname{cov}\left(\hat{\beta}_{N}^{(2)}\right) \leq \operatorname{cov}\left(\hat{\beta}_{N}^{(1)}\right)$. Then, $\operatorname{cov}\left(\hat{\gamma}_{N}^{(2)}\right) \leq \operatorname{cov}\left(\hat{\gamma}_{N}^{(1)}\right)$.

Proof. First let us comment that, in the context of this paper, $\operatorname{cov}\left(\hat{\beta}_{N}^{(2)}\right) \leq$ $\operatorname{cov}\left(\hat{\beta}_{N}^{(1)}\right)$ typically occurs because the subvector $\beta$ apears in the added term $G_{m+1}(z) u_{m+1}(t)$ of experiment $X_{2}$. Let $\alpha^{(1)}$ be the vector of all parameters, other than $\beta$, that appear in all other transfer models in experiment $X_{1}$, and let $\alpha^{(2)}$ be the vector of all such parameters in experiment $X_{2}$. Then the 
information matrices for the two experiments have the following forms:

$$
M^{(i)}=\left(\begin{array}{ccc}
M_{\alpha^{(i)} \alpha^{(i)}} & M_{\alpha^{(i)} \beta} & 0 \\
M_{\beta \alpha^{(i)}} & M_{\beta \beta} & M_{\beta \gamma} \\
0 & M_{\gamma \beta} & M_{\gamma \gamma}
\end{array}\right) \quad i=1,2
$$

Note that $\alpha^{(1)}$ and $\alpha^{(2)}$ (and hence $M_{\alpha^{(1)} \alpha^{(1)}}$ and $M_{\alpha^{(2)} \alpha^{(2)}}$ ) do not necessarily have the same dimension; typically $\operatorname{dim}\left(\alpha^{(2)}\right) \geq \operatorname{dim}\left(\alpha^{(1)}\right)$ when experiment $X_{2}$ contains an additional input term. Let us denote by $C^{(i)}$ the inverse of $M^{(i)}$, with an identical partitioning. The $(2,2)$ and $(3,3)$ block-elements of $C^{(i)}$ are then obtained as:

$$
\begin{aligned}
C_{\beta \beta}^{(i)} & =\left[M_{\beta \beta}-M_{\beta \gamma} M_{\gamma \gamma}^{-1} M_{\gamma \beta}-M_{\beta \alpha^{(i)}} M_{\alpha^{(i)} \alpha^{(i)}}^{-1} M_{\alpha^{(i)} \beta}\right]^{-1} \\
C_{\gamma \gamma}^{(i)} & =\left[M_{\gamma \gamma}-M_{\gamma \beta}\left(M_{\beta \beta}-M_{\beta \alpha^{(i)}} M_{\alpha^{(i)} \alpha^{(i)}}^{-1} M_{\alpha^{(i)} \beta}\right)^{-1} M_{\beta \gamma}\right]^{-1}
\end{aligned}
$$

Combining these last two equations, we get a remarkable relationship between $C_{\gamma \gamma}^{(i)}$ and $C_{\beta \beta}^{(i)}$ in which the dependence on the accuracy of the estimates of all other parameters (those contained in $\alpha^{(i)}$ ) has been eliminated:

$$
C_{\gamma \gamma}^{(i)}=\left[M_{\gamma \gamma}-M_{\gamma \beta}\left(\left(C_{\beta \beta}^{(i)}\right)^{-1}+M_{\beta \gamma} M_{\gamma \gamma}^{-1} M_{\gamma \beta}\right)^{-1} M_{\beta \gamma}\right]^{-1} .
$$

This relationship holds for both experiments, $X_{1}$ and $X_{2}$. Since $\operatorname{cov}\left(\hat{\beta}_{N}^{(i)}\right)=$ $\frac{\sigma_{e}^{2}}{N} C_{\beta \beta}^{(i)}$ and $\operatorname{cov}\left(\hat{\gamma}_{N}^{(i)}\right)=\frac{\sigma_{e}^{2}}{N} C_{\gamma \gamma}^{(i)}$ we have thus proved that

$$
\operatorname{cov}\left(\hat{\beta}_{N}^{(2)}\right) \leq \operatorname{cov}\left(\hat{\beta}_{N}^{(1)}\right) \Leftrightarrow \operatorname{cov}\left(\hat{\gamma}_{N}^{(2)}\right) \leq \operatorname{cov}\left(\hat{\gamma}_{N}^{(1)}\right) .
$$

Observe that the result of Lemma 2 is rather general and applies to broader situations than the one considered here of the addition of an input signal term. Suppose, for example, that the accuracy of some subvector $\hat{\beta}_{N}$ appearing in one of the transfer function models is improved because the level of the input signal power is increased at the corresponding input. Then, the covariance of the estimated parameters appearing in other transfer function models jointly with the $\beta$ subvector is also decreased.

With the results of the two previous lemmas, we can now state precisely which of the parameter estimates of a MISO system can be improved by the addition of a new input term. To make a precise statement, we need to introduce the following definition. 
Definition 2 Consider a set of parametrized transfer functions $\mathcal{S}=$ $\left\{G_{1}, G_{2}, \ldots, G_{m}\right\}$. We shall say that $G_{i}$ is linked with $G_{j}$ if $G_{i}$ and $G_{j}$ have common parameters; we denote this by $G_{i} \cap G_{j}$. We shall say that $G_{i}$ is connected to $G_{j}$ if there exists a sequence of elements of $\mathcal{S}$ such that $G_{i} \cap G_{k} \cap \ldots \cap G_{l} \cap G_{j}$.

We can then state the main result of this paper.

Theorem 3 Consider an identification experiment with the m-input 1-output system described by (13), and consider now a second identification experiment with the same input signals $u_{1}, \ldots, u_{m}$, but with the additional input term $G_{m+1}(z) u_{m+1}(t)$. Let the Standing Assumptions hold in both experiments. Then, the addition of the input term $G_{m+1}(z) u_{m+1}(t)$ in the second experiment improves the parameter estimates of all polynomials in all transfer function models $G_{i}, i=1, \ldots, m$ and $H$ that are connected to the model of $G_{m+1}(z)$.

Proof. The proof is an immediate consequence of Lemmas 1 and 2.

With the results of this section, we are now able to provide an answer to the quizz proposed in the Introduction. With the Standing Assumptions holding, the presence of a non-zero input signal $u_{2}$ in the four model structures, compared to the situation where $u_{2}=0$, improves the quality of the following polynomial estimates: for model $M_{1}: A, B_{1}$ and $C$; for model $M_{2}: B_{1}, F$ and $D$; for model $M_{3}: B$ and $F_{1}$; and for model $M_{4}: C$ and $D$.

\section{$5 \quad$ Input design considerations}

In this section, we come back to the initial motivation that led us to investigate the variance formulas presented so far: assuming that we are to identify a system with several inputs, is it better to excite one input at a time or all inputs simultaneously, given that these inputs are independent? We consider systems with two inputs, since all qualitative statements we make in this section will remain unchanged for systems with more than two inputs. In order to make fair comparisons, and since the variance of all parameter estimates decays as $\frac{1}{N}$, we assume that the total experimentation time $N$ is fixed. Thus, we compare excitation with two independent signals $u_{1}$ and $u_{2}$ simultaneously during the whole time interval, with excitation with $u_{1}$ only over $\left[0, \frac{N}{2}\right]$ and with $u_{2}$ only over $\left[\frac{N}{2}, N\right]$, say. Finally, we shall limit our analysis to the model structures that are most commonly used in system identification, and we also consider that the Standing Assumptions hold for the signals $u_{1}$ and $u_{2}$. 


\subsection{ARX and ARMAX model structures}

We first consider the input design question stated above for model structures that have common parameters in the input/output and noise models, i.e. ARX and ARMAX models. For these model structures, we have shown that the addition of a new input signal contributes to a strict decrease of the covariance of all estimated polynomials. We give a complete analysis for the case of an ARX model structure (see subsection 3.2).

In the case of simultaneous excitation over $[0, N]$, the information matrix is given by (see (19)):

$$
M_{s i m}=\left(\begin{array}{ccc}
R_{Y Y}(e)+R_{Y Y}\left(u_{1}\right)+R_{Y Y}\left(u_{2}\right) & R_{Y U_{1}} & R_{Y U_{2}} \\
R_{U_{1} Y} & R_{U_{1} U_{1}} & 0 \\
R_{U_{2} Y} & 0 & R_{U_{2} U_{2}}
\end{array}\right)
$$

In the case of separate excitation over half the interval length, and assuming that the power spectra (or, equivalently, the covariances) of the excitation signals are identical in both experiments, we get:

$$
M_{\text {sep }}=\left(\begin{array}{ccc}
R_{Y Y}(e)+\frac{1}{2} R_{Y Y}\left(u_{1}\right)+\frac{1}{2} R_{Y Y}\left(u_{2}\right) & \frac{1}{2} R_{Y U_{1}} & \frac{1}{2} R_{Y U_{2}} \\
\frac{1}{2} R_{U_{1} Y} & \frac{1}{2} R_{U_{1} U_{1}} & 0 \\
\frac{1}{2} R_{U_{2} Y} & 0 & \frac{1}{2} R_{U_{2} U_{2}}
\end{array}\right)
$$

Now observe that

$$
M_{\text {sim }}-M_{\text {sep }}=\frac{1}{2}\left(\begin{array}{ccc}
R_{Y Y}\left(u_{1}\right)+R_{Y Y}\left(u_{2}\right) & R_{Y U_{1}} & R_{Y U_{2}} \\
R_{U_{1} Y} & R_{U_{1} U_{1}} & 0 \\
R_{U_{2} Y} & 0 & R_{U_{2} U_{2}}
\end{array}\right)>0,
$$

where the positive definiteness follows from the Standing Assumptions on the signals $u_{1}$ and $u_{2}$. Therefore $\left(M_{\text {sim }}\right)^{-1}<\left(M_{\text {sep }}\right)^{-1}$ and, using (11), it follows that the covariances of $\widehat{\mathbf{a}}$ and $\widehat{\mathbf{b}_{1}}$ are both strictly larger in the case of separate excitation. The expressions of these covariances follow easily from (49) and (50). For the case of simultaneous excitation over $[0, N]$, we get:

$$
\begin{aligned}
{[\operatorname{cov}(\widehat{\mathbf{a}})]^{-1} } & =\frac{N}{\sigma_{e}^{2}}\left[R_{Y Y}(e)+\Gamma\left(u_{1}\right)+\Gamma\left(u_{2}\right)\right] \\
{\left[\operatorname{cov}\left(\widehat{\mathbf{b}_{1}}\right)\right]^{-1} } & =\frac{N}{\sigma_{e}^{2}}\left[R_{U_{1} U_{1}}-R_{U_{1} Y}\left(R_{Y Y}(e)+R_{Y Y}\left(u_{1}\right)+\Gamma\left(u_{2}\right)\right)^{-1} R_{Y U_{1}}\right]
\end{aligned}
$$


where we have introduced the matrices $\Gamma\left(u_{i}\right) \triangleq R_{Y Y}\left(u_{i}\right)-R_{Y U_{i}} R_{U_{i} U_{i}}^{-1} R_{U_{i} Y}, i=$ 1,2 , which are positive definite by the Standing Assumptions. In the case of separate excitation over half the interval length, we get:

$$
\begin{aligned}
{[\operatorname{cov}(\widehat{\mathbf{a}})]^{-1} } & =\frac{N}{2 \sigma_{e}^{2}}\left[R_{Y Y}(e)+\Gamma\left(u_{1}\right)\right]+\frac{N}{2 \sigma_{e}^{2}}\left[R_{Y Y}(e)+\Gamma\left(u_{2}\right)\right] \\
& =\frac{N}{\sigma_{e}^{2}}\left[R_{Y Y}(e)+\frac{1}{2}\left(\Gamma\left(u_{1}\right)+\Gamma\left(u_{2}\right)\right)\right] \\
{\left[\operatorname{cov}\left(\widehat{\mathbf{b}_{1}}\right)\right]^{-1} } & =\frac{N}{2 \sigma_{e}^{2}}\left[R_{U_{1} U_{1}}-\frac{1}{2} R_{U_{1} Y}\left(R_{Y Y}(e)+\frac{1}{2} R_{Y Y}\left(u_{1}\right)+\frac{1}{2} \Gamma\left(u_{2}\right)\right)^{-1} R_{Y U_{1}}\right]
\end{aligned}
$$

In the case where the output power is constrained, it is reasonable to translate the constraint on the output power into the constraint $R_{Y Y} \leq R_{\max }$ for some given $R_{\max }$. In the case of simultaneous excitation, the information matrix is then again given by $(49)$, where the $(1,1)$ block-term is now equal to $R_{\max }$. In the case of separate excitation, one should then adjust the power level of the input signal $u_{1}$ so that, during the interval $\left[0, \frac{N}{2}\right]$ during which it is applied, it produces the same output power (or the same $R_{\max }$ ) as in the simultaneous excitation experiment, and likewise for $u_{2}$ during the interval $\left[\frac{N}{2}, N\right]$. Although an exact comparison between the covariances achieved with simultaneous and with separate excitation is very difficult to compute, a reasonable approximation shows that the information matrix will be nearly the same as for the case of simultaneous excitation. Thus, in the case of output power constraints, the two strategies will yield roughly the same accuracy for the parameter estimates.

We conclude this subsection with an additional input design recommendation that results from the analysis of Section 3. Suppose we are to identify a SISO ARX or ARMAX model, and assume that the power of the input signal $u$ is constrained. If it is physically possible to add an additional excitation $u_{2}$ such that the input-output model for $u_{2}$ has common parameters with the model for $u$ or with the noise model, then our analysis suggests that adding this second excitation improves the accuracy of the estimates of the other polynomials. In particular, if one needs to identify an ARX model $A y=B u+e$ with a power constraint on $u$ and if it is possible to add a second input signal such that $A y=B u+B_{2} u_{2}+e$, then the excitation by $u_{2}$ will improve the parameter estimates of $A$ and $B$.

\subsection{FIR, OE and BJ model structures}

We consider now separately parametrized model structures, i.e. FIR, OE and BJ models. For such model structures, the input $u_{i}$ affects the precision of the 
estimate of $G_{i}(z)$ only. If there is no constraint on the output variance, the optimal design is then to excite both inputs simultaneously and independently during the whole time interval of length $N$, with the maximum admissible input signal powers. If there is a constraint on the output power, and assuming for simplicity that the contributions to the output power of the two input signals are the same, then calculations show that exciting the two inputs simultaneously during the whole interval, or exciting each separately at the maximum allowable power during half of the total experimentation time will lead to the same covariance for the parameters of the estimated input/output transfer functions.

\section{Conclusions}

We have examined the effect of an additional input signal excitation on the variance of the parameter estimates of multiple-input single-output systems, in the situation where the system is in the model set. We have shown that the addition of an input term can only improve the accuracy of some of the other estimated transfer functions. More precisely, we have presented conditions on the structure of the parametrization of MISO systems that allow one to know exactly which transfer functions will be estimated more accurately when a new input term is added. We have also shown that, if the accuracy of some of the parameters of a transfer function model is improved, then the accuracy of all parameters in this transfer function model is improved.

For the commonly used ARX and ARMAX model structures, the addition of a new input signal $u_{m+1}$ improves the accuracy of all parameter estimates, including those of the $B_{i}$ polynomials corresponding to the other signals $u_{i}, i=$ $1, \cdots, m$, and of the $C$ polynomial of the noise model. For FIR, OE, or BJ structures, the additional input signal $u_{m+1}$ does not affect the variances of the parameters corresponding to the other inputs or to the noise model.

Finally, our results have enabled us to address the question of whether one should excite the different inputs of MISO systems simultaneously or separately. In a nutshell, the general recommendation is that, by applying simultaneous and independent excitations, one can never do worse than with separate excitation, and one can often do much better.

\section{References}

[1] X. Bombois, M. Gevers, and G. Scorletti. Open-loop versus closed-loop identification of box-jenkins models: a new variance analysis. In submitted to 
joint CDC-ECC 2005, Seville, Spain, December 2005.

[2] B. S. Dayal and J. F. MacGregor. Multi-output process identification. Journal of Process Control, 7(4):269-282, 1997.

[3] S. Garatti, M.C. Campi, and S. Bittanti. Assessing the quality of identified models through the asymptotic theory - when is the result reliable? Automatica, 40(8):1319-1332, 2004.

[4] A.A.B. Klein and G. Mélard. The information matrix of multiple-input singleoutput time series models. Journal of Computational and Applied Mathematics, 51:349-356, 1994.

[5] L. Ljung. Asymptotic variance expressions for identified black-box transfer function models. IEEE Trans. Automatic Control, AC-30:834-844, 1985.

[6] L. Ljung. System Identification: Theory for the User, 2nd Edition. PrenticeHall, Englewood Cliffs, NJ, 1999.

[7] B. Ninness and H. Hjalmarsson. Variance error quantifications that are exact for finite model order. IEEE Transactions on Automatic Control, 49(8):1275-1291, August 2004.

[8] T. Söderström and P. Stoica. System Identification. Prentice-Hall International, Hemel Hempstead, Hertfordshire, 1989.

[9] L.L. Xie and L. Ljung. Asymptotic variance expressions for estimated frequency functions. IEEE Transactions on Automatic Control, AC-46:1887-1899, 2001.

[10] Y. C. Zhu. Multivariable process identification for MPC: the asymptotic method and its applications. Journal of Process Control, 8(2):101-115, 1998.

[11] Y. C. Zhu and T. Backx. Identification of multivariable industrial processes for simulation, diagnosis and control. In M. J. Grimble and M. A. Johnson, editors, Advances in Industrial Control. Springer-Verlag, London, 1993. 